\section{Hematological, hepatic, and renal adverse effects of high dose methotrexate injection (50 mg weekly) in severe psoriasis cases}

\section{Adissa Tiara Yulinvia, \\ Prasta Bayu Putra, Niken Trisnowati, Sunardi Radiono}

Department of Dermatology and Venereology, Faculty of Medicine, Public Health, and Nursing, Universitas Gadjah Mada, Yogyakarta, Indonesia

\begin{abstract}
Ten to $30 \mathrm{mg}$ weekly methotrexate (MTX) has long been used as a treatment for severe, recalcitrant psoriasis. However, some patients do not improve with those dose and there has been oral methotrexate scarcity period in Indonesia since 2015. This is the first study to evaluate the safety profile of high dose (50 mg weekly) MTX injection in severe psoriasis cases at Sardjito hospital, Yogyakarta. We conducted a retrospective adverse effects evaluation from demographic and laboratory data of 23 severe psoriasis patients who had received six weekly intramuscular injections of MTX. For the results, five $(21.70 \%)$ patients developed hematological adverse effects, four (17.30\%) patients developed hepatic adverse effects, and three (13.05\%) patients developed renal adverse effects. Majority of the abnormal laboratory findings in this study were mild and transient. High dose MTX injection appears to be safe for severe psoriasis if proper patient baseline condition assessment was taken before starting therapy, all contraindications have been excluded, and is used under close supervision at high level medical facilities.
\end{abstract}

\section{Introduction}

Methotrexate (MTX) inhibits dihydrofolate reductase and has been used as a treatment for severe, recalcitrant psoriasis for more than 50 years. MTX has serious fatal side effects such as bone marrow suppression, liver cirrhosis, and pulmonary disease that cause the limitation of its use. However, its side effects are reported fewer and milder with injection routes. The recommended dose from majority guidelines are ranged from $10-30 \mathrm{mg}$ weekly because of concerns about its toxicity. ${ }^{1,2}$

MTX dosage prescribed in Asian countries is at least $25 \%$ lower than European standard. This might result from studies finding that MTX treatment in Asia is correlated with higher incidence of adverse reactions: more than 50\% psoriasis patients receiving MTX treatment in psoriasis center in Malaysia developed abnormal liver function, and $10 \%$ patients had to stop the treatment because of drug hepatotoxicity. Other important aspect of this dosages difference is the difference in mean Body Mass Index (BMI) score between United State, Europe and Asia $\left(29,22.5\right.$, and $23.7 \mathrm{~kg} / \mathrm{m}^{2}$, respectively). 3,4 Some severe psoriasis cases do not improve with the recommended doses in guidelines, and in Indonesia, specifically in Yogyakarta, there has been a period of oral methotrexate scarcity since 2015. In Sardjito hospital, the prevalence of severe psoriasis patients who need high MTX dose from 2015 to 2017 was 129 from total 612 psoriasis patients, it means that a significant number of patients could not get the treatment they needed. To overcome this condition, in Sardjito hospital, severe and recalcitrant psoriasis patients were treated with high dose MTX injection, $50 \mathrm{mg}$ intramuscular weekly. The objective of this study was to evaluate the safety profiles of weekly high dose MTX injection in severe psoriasis patients.

\section{Materials and Methods}

This is a retrospective study of consecutive patients diagnosed with severe, recalcitrant psoriasis who were admitted to Dermatology and Venereology ward at Sardjito Hospital, Yogyakarta for MTX injection (50 mg weekly) from July to December 2017. Patients were included in this study if they had a diagnosis of severe psoriasis according to the published criteria, were given six weekly $50 \mathrm{mg}$ MTX injection, had complete laboratory records, and had signed the informed consent form. The following data were collected at baseline: age, sex, BMI, other conditions which consisted of comorbid diseases, concomitant drug used, alcoholic abuse, and folic acid supplementation. Complete blood count $(\mathrm{CBC})$, liver function and renal function tests were conducted at baseline (week 0) and one week after each MTX injection.

Adverse effects were defined as abnormal laboratory finding of complete blood count results (hemoglobin, leukocyte and thrombocyte counts), liver function test and renal function test during MTX injection.

\section{Results}

Total 23 patients with severe, recalcitrant psoriasis were included in this study.
Correspondence: Adissa Tiara Yulinvia, Department of Dermatology and Venereology, Faculty of Medicine, Public Health and Nursing, Universitas Gadjah Mada, Farmako Sekip Utara road, Sinduadi, Mlati, Sleman, Yogyakarta Province, Indonesia 55281.

Tel.: +62 (274) 560700

E-mail: adissatiara@gmail.com

Key words: psoriasis, methotrexate, $50 \mathrm{mg}$, injection, adverse effect.

Acknowledgements: We would like to thank Tuntas Rayinda, MD and Schandra Purnamawati, MD for the advices during the making of this paper; Vika Fintaru, MD, Jesslyn Amelia, MD, Januar Devita Sari, MD, and Danar Wicaksono, MD for the assistance with data collection of this paper.

Contributions: ATY, PBP, NT, SR study conception and design; ATY, PBP data collection, analyses, writing drafts of article; NT, SR supervisor, critical revision, final approval.

Conflict of interest: the authors declare no conflict of interest.

Received for publication: 1 February 2019. Accepted for publication: 13 February 2019.

This work is licensed under a Creative Commons Attribution-NonCommercial 4.0 International License (CC BY-NC 4.0).

(C) Copyright et al., 2019

Licensee PAGEPress, Italy

Dermatology Reports 2019; 11(s1):

doi:10.4081/dr.2019.8053

Sixteen patients $(69.60 \%)$ were male and 7 $(30.40 \%)$ were female. According to $\mathrm{WHO}$ age classification, ten $(43.50 \%)$ patients were young-adult age group, 7 (30.40\%) were middle-aged group, and $6(26.10 \%)$ were elderly group. According to WHO BMI classification, $3(13.05 \%)$ patients were underweight, 10 (43.50\%) were normal weight, $7(30.40 \%)$ patients were overweight, and 3 patients $(13.05 \%)$ were obese. Four $(17.40 \%)$ patients had diabetes mellitus (DM); none had liver disease, alcohol abuse nor consumption of drugs that interacted with MTX, and all patients had received folic acid supplementation. The demographic characteristics of study participants are shown in Table 1.

Patients with at least one abnormal laboratory parameter result were included as having adverse effect. For hematological adverse effect, $5(21.70 \%)$ patients developed mild anemia (Hb level 9-12.9 g/dL); of those, one patient $(4.30 \%)$ also developed thrombocytopenia (platelet count $\left.<150 \times 10^{3} / \mu \mathrm{L}\right)$ and one (4.30\%) patient also 
developed thrombocytopenia and leukopenia (leukocyte count $\left.<4.5 \times 10^{3} / \mu \mathrm{L}\right) .^{5}$ For laboratory standards of liver function test, the upper normal limit (UNL) for serum SGOT and SGPT were $32 \mathrm{U} / \mathrm{L}$ and $33 \mathrm{U} / \mathrm{L}$, respectively. In this study, 3 patients $(13.10 \%)$ developed mild SGOT level elevation $(<5 \mathrm{UNL})$; of those, 2 patients $(8.70 \%)$ also had moderate SGPT level elevation (5-10 x UNL), and 1 patient (4.30\%) also had mild SGPT level elevation $(<5$ UNL). One patient (4.30\%) only developed mild SGPT level elevation. ${ }^{6}$ Three patients (13.05\%) developed abnormal renal function test. Two patients $(8.70 \%)$ had elevated creatinine serum level, and one patient $(4.30 \%)$ had elevated BUN level. The hematological, hepatic, and renal adverse effects were summarized in Table 2.

Table 1. Demographic characteristics of study participants.

\begin{tabular}{lc} 
Characteristics & $\begin{array}{c}\text { Patients (\%) } \\
\mathrm{n}=23\end{array}$ \\
Sex & \\
Male & $16(69.60)$ \\
Female & $7(30.40)$ \\
Age $^{\mathrm{a}}$ & \\
Young-adult $(18-44$ years-old) & $10(43.50)$ \\
Middle-aged $(45-59$ years-old) & $7(30.40)$ \\
Elderly $(\geq 60$ years-old) & $6(26.10)$ \\
\hline Body Mass Index (kg/m²) & \\
Underweight $(<18.5)$ & $3(13.05)$ \\
Normal $(18.5-22.9)$ & $10(43.50)$ \\
Overweight $(23-24.9)$ & $7(30.40)$ \\
Obese $(>28)$ & $3(13.05)$ \\
Other conditions & \\
Diabetes Mellitus & $4(17.40)$ \\
Liver diseases & $0(0.00)$ \\
Alcohol abuse & $0(0.00)$ \\
Consumption of drugs which & $0(0.00)$ \\
interact with MTX & \\
Folic acid supplementation & $23(100.00)$ \\
\hline awHO age classification; bwHo Body Mass Index classification.
\end{tabular}

Table 2. Frequency of hematologic, hepatic, and renal adverse effects.

Measurement $\begin{gathered}\text { Number } \\ \text { of participants } \\ (\%) \\ \mathrm{n}=23\end{gathered}$

Mild anemia $(9-11.9 \mathrm{~g} / \mathrm{dL}) \quad 5(21.70)$

Thrombocytopenia $\left(<150 \times 10^{3} / \mu \mathrm{L}\right) \quad 2(8.70)$

Leukopenia $\left(<4.5 \times 10^{3} / \mu \mathrm{L}\right) \quad 1(4.30)$

Mild SGPT elevation $(<5$ UNL) $\quad 2(8.70)$

Moderate SGPT elevation (5-10 UNL)2 (8.70)

Mild SGOT elevation $(<5$ UNL) $3(13.05)$

Elevated BUN level $(>20 \mathrm{mg} / \mathrm{dL}) \quad 1(4.30)$

Elevated creatinine level (>1 mg/dL)2 (8.70)

SGOT, Serum Glutamic Oxaloacetic Transaminase; SGPT, Serum Glutamic Pyruvic Transaminase; BUN, Blood Urea Nitrogen.

\section{Discussion}

MTX is known for its side effects potential, with the most frequent is abnormal liver function tests. Hepatotoxicity is a common long-term problem, and bone marrow toxicity being the most serious shortterm side effect of MTX. Other reported systemic adverse effects are nephrotoxicity, pulmonary fibrosis, and mucocutaneous reactions. Maryann et al (2004) concluded that MTX treatment in general recommended dose for psoriasis is safe for short term use $(<1-2$ years $)$. $^{2,7}$

Magdalena et al (2014) stated that adverse symptoms of MTX occur with varying severity, posing a tendency to subside after dose reduction or treatment discontinuation. For laboratory test results, the most common deviations were found in liver tests and blood elements. Leukopenia and thrombocytopenia usually occur between 7 and 10 days of treatment, but they can occur at any stage of treatment. ${ }^{3}$

MTX can suppress hematopoiesis process and causes anemia, leukopenia, thrombocytopenia, or pancytopenia. In this study, decrease in hemoglobin level below lower normal limit was found in 5 patients (in two cases the anemic state was transient, and in three cases the condition persists until after the sixth injection). Leukopenia was seen in $1(4.30 \%)$ patient, and thrombocytopenia in $2(8.70 \%)$ patients.

MTX can cause acute (elevated transaminases) and chronic (fibrosis and cirrhosis) hepatotoxicity. An increase in liver enzyme to more than five times upper normal limit was found in two patients (one case after the second injection and one case after the fifth injection), in both cases, delaying the injection and curcuma supplementation three times a day for 1-2 days caused the normalization of their liver function tests.

The aim of folic acid supplementation is to lessen hepatotoxicity and prevent hematopoietic system adverse reactions. The risk factors for developing abnormal liver function test are (i) history of past or current alcohol intake ( $\geq 2$ drink per day), (ii) diabetes, (iii) obesity, (iv) exposure history of hepatotoxic drugs, and (v) advanced age. Liver chemistry tests should be obtained at least a week interval after MTX last dose because liver chemistry values are frequently elevated 1 to 2 days after MTX treatment. Maximum myelosuppression effect commonly occurs within 7 to 10 days after MTX treatment. ${ }^{3,8}$

Acute renal failure induced by MTX is a rare but serious side effect. This is caused by precipitation of MTX or its metabolites in renal tubules causing obstruction and diminution of renal clearance. In this study, 2 patients $(8.70 \%)$ had transient elevated creatinine serum level, and one patient $(4.30 \%)$ had transient elevated BUN level. The solubility of MTX and its metabolites is greater in urine with higher $\mathrm{pH}$. Therefore, monitoring renal function before, during, and after MTX injection is recommended. Urine alkalinization and leucoverin are the mainstay in the management of early renal dysfunction signs. ${ }^{9}$

MTX treatment should be delayed until recovery if: (i) leukocyte count less than $1.5 \times 10^{3} / \mu 1$; (ii) neutrophil count less than $200 / \mu 1$ ); (iii) platelet count less than $75 \times 10^{3} / \mu 1$ ); (iv) SGPT level more than 450 $\mathrm{U} / \mathrm{l}$; (v) mucositis and persistent pleural effusion. ${ }^{8}$ All those criteria were not found in our subjects.

Our subjects represent a group of severe psoriasis patients treated with six weekly intramuscular injections of high dose MTX at Sardjito hospital, Yogyakarta. The MTX dosage used in this study was $50 \mathrm{mg}$ weekly, and with this treatment, marked clinical improvement was observed in most of the patients, but unfortunately this treatment efficacy was not evaluated in this study. To date, this is the first study that specifically deal with the short-term safety profile of high dose MTX injection in patients with severe psoriasis in Indonesia. ${ }^{10,11}$

The results of our study were limited because of its small sample size and retrospective nature. This study had no control group and no clinical efficacy data. Larger cohorts of severe psoriasis patients with longer study time are needed to finally establish the safety profile of this dose drug.

\section{Conclusions}

High dose MTX injection (50 mg weekly) appears to be a safe drug if proper patient baseline condition assessment was taken before therapy, all contraindications have been excluded, and is used under close supervision in high level medical facilities.

\section{References}

1. Negrei C, Boda D. The role of methotrexate in psoriatic therapy in the age of biologic and biosimilar medication: therapeutic benefits versus toxicology emergencies. Psoriasis Anca Chiriac, IntechOpen 2017. Available from: https://www.intechopen. com/ books/an-interdisciplinary-approach-topsoriasis/the-role-of-methotrexate-inpsoriatic-therapy-in-the-age-of-biologic-and-biosimilar-medication 
2. Mikhail M, Scheinfeld NS. Therapy treatment options for psoriasis: topical and systemic. Therapy 2004;1:319-30.

3. Czarnecka-Operacz M, SadowskaPrzytocka A. The possibilities and principles of methotrexate treatment of psoriasis - The updated knowledge. Postep Derm Alergol 2014;31: 392-400.

4. Weidmann A, Foulkes AC, Kirkham N, Reynolds NJ. Methotrexate toxicity during treatment of chronic plaque psoriasis: a case report and review of the literature. Dermatol Ther 2014;4: 14556.
5. Dubey L, Chatterjee S, Ghosh A. Hepatic and hematological adverse effects of long-term low-dose methotrexate therapy in rheumatoid arthritis: an observational study. Indian J Pharmacol 2016;48:591-4.

6. Giannini EG, Testa R, Savarino V. Liver enzyme alteration: a guide for clinicians. Can Med Assoc J 2005;172:36779.

7. Dahl MGC, Gregory MM, Scheuer PJ. Methotrexate hepatotoxicity in psoriasis - comparison of different dose regimens. Brit Med J 1972;1:654-6.
8. Roenigk HH, Auerbach R, Maibach HI, Weinstein GD. Methotrexate in psoriasis: revised guidelines. J Am Acad Dermatol 1988;19:145-56.

9. Gaies E, Jebabli N, Trabelsi S, et al. Methotrexate side effects : review article. J Drug Metab Toxicol 2012;3:1-5.

10. Hirshberg B, Muszkat M, Schlesinger O, Rubinow A. Safety of low dose methotrexate in elderly patients with rheumatoid arthritis. Postgrad Med J 2000;76:787-9. 